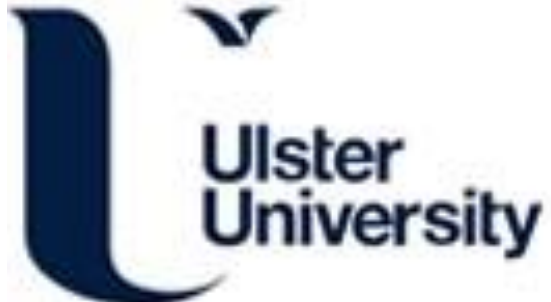

\section{Sensor-based Vital Sign Monitoring, Analysis and Visualisation for Ageing in Place}

Kerr, E., Coleman, SA., Kerr, D., Vance, P., Gardiner, B., McGinnity, TM., Zhang, Y., Fei, W., \& Wu, C.

(Accepted/In press). Sensor-based Vital Sign Monitoring, Analysis and Visualisation for Ageing in Place. In Unknown Host Publication IEEE.

Link to publication record in Ulster University Research Portal

\section{Published in:}

Unknown Host Publication

\section{Publication Status:}

Accepted/In press: 14/03/2018

\section{Document Version \\ Author Accepted version}

\section{General rights}

Copyright for the publications made accessible via Ulster University's Research Portal is retained by the author(s) and / or other copyright owners and it is a condition of accessing these publications that users recognise and abide by the legal requirements associated with these rights.

\section{Take down policy}

The Research Portal is Ulster University's institutional repository that provides access to Ulster's research outputs. Every effort has been made to ensure that content in the Research Portal does not infringe any person's rights, or applicable UK laws. If you discover content in the Research Portal that you believe breaches copyright or violates any law, please contact pure-support@ulster.ac.uk. 


\section{Sensor-based Vital Sign Monitoring, Analysis and Visualisation for Ageing in Place}

\author{
E. P. Kerr, S.A. Coleman, D. Kerr, P. \\ Vance, B. Gardiner \\ School of Computing and Intelligent \\ Systems, \\ Ulster University, N. Ireland, UK \\ email: \{ep.kerr, sa.coleman, d.kerr, \\ p.vance, b.gardiner\}@ulster.ac.uk
}

\author{
Y. Zhang, F. Wang and C. Wu \\ Faculty of Robot Science and \\ Engineering, Northeastern University, \\ Shenyang, China. \\ email: \{zhangyunzhou, wangfei, \\ wuchengdong\}@mail.neu.edu.cn
}

\begin{abstract}
With the ever-increasing global population and average life expectancy, care homes and care at home services are continuously being stretched beyond capacity. Recent developments in tactile sensing have enabled robot systems to measure human vital signs such as beats per minute (BPM), Respiratory Rate (RR) and Capillary Refill Time (CRT). Using robotic systems to measure vital sign data in the home of an elderly or disabled person would greatly assist medical and health services. This paper proposes the use of a vital sign measuring robotic system together with Cloud computing to intelligently process big data and ascertain the current health status of the service user without the need to expose their identity or burden health professionals. Furthermore, a method that enables medical professionals to visualise the data for a complete geographical region as well as for individual patients is presented and hence we provide details of a closed loop system to support ageing-in-place.
\end{abstract}

Keywords-tactile sensing, cloud computing, data visualisation, health status

\section{INTRODUCTION}

The global population is continuously increasing together with the average life expectancy. According to the World Health Organisation [1] most people can expect to live to 60 years and beyond. By 2050, the world's population aged 60 years and older is expected to total 2 billion, up from 900 million in 2015. Today, 125 million people globally are aged 80 years or older. In China, the number of older persons is projected to grow by $71 \%$ between 2015 and 2030 and by 2050, it is estimated there will be 120 million people aged 80 years or older in China alone, and 434 million people in the 60+ years age group worldwide [2]. By 2050, $80 \%$ of all older people will live in low- and middle-income countries. Places such as Brazil, China and India will have slightly more than 20 years to make necessary adaptation and by 2050 Chile, China, the Islamic Republic of Iran and the Russian Federation will have a similar proportion of older people to Japan. Population ageing and growth in the number of persons at very advanced ages, in particular, puts pressure on health systems, increasing the demand for care, services and technologies to prevent and treat non-communicable diseases and chronic conditions associated with old age.

Timely, informative and appropriate responses to medical emergencies in an aged person's home are essential to ensure the health and well-being of this increasing subset of the population who often live independently and by themselves. With this, there is a need to offer the reassurance of responsive medical care should a critical situation arise. With the increase and promotion of robotic aid to support and assist daily living then it would be only reasonable to include the ability to offer support in medical emergencies - yet this aspect of assistance is often overlooked. There have been numerous assistive technologies developed to help with nursing elderly people particularly with the manual handling aspect of moving patients etc., [3] or to enable a doctor to carry out basic remote assessments of a patient's health [4]. However, in order to complete an accurate assessment of a patient's vital signs it is reported that these systems need much more advanced tactile sensing capabilities [4].

Vital sign monitoring is typically a task that is performed by humans in clinical or emergency situations or sometimes even in a domestic environment. Pulse may be measured at various positions on the body by a person pressing firmly on an artery which is located close to the skin whilst counting the beats per minute (BPM). Generally, this requires some training and experience in order to obtain accurate estimations. Respiration rate (RR) may also be measured by determining the number of breaths taken per minute by counting how many times the chest rises in this period. Capillary Refill Time (CRT) is the time taken for the colour to restore in an external capillary bed following blanching caused by pressure being applied by pressing on the fingernail, toenail, soft tissue at the kneecap or forearm, centre of the chest or on the forehead [5]. The forehead is usually the most accessible area to access in a medical emergency or disaster situation considering the individuals will most likely be wearing clothes. When measured at the forehead, the normal time for CRT should be 
less than 2 seconds for an adult, up to 3 seconds for an infant and up to 4.5 seconds for an elderly person [6].

A number of devices exist for measuring a range of vital signs. The Scanadu Scout is a portable electronic device for consumer use designed to measure different physiological parameters, including temperature, heart rate, blood oxygenation, respiratory rate, electrocardiogram (ECG), and diastolic/systolic blood pressure. The lightweight device is marketed as being able to return key vital sign results in less than 10 seconds by placing it in contact with a patient's temple. The device is currently promoted through crowdfunding and is not yet publicly available. It relies on a person being present to apply the device. The Kito (previously known as Wello) by Azoi is an iPhone cover with built-in circuitry and sensors that is able to track a variety of vital signs such as blood pressure, heart rate, blood oxygen level, body temperature and ECG. Again, this device is currently not available for public use. Other simpler devices are available, for example the Vital Signs Camera app from Philips performs a contactless measurement of heart rate and breathing rate using a smartphone camera. Sotera Wireless have developed a vital sign monitoring platform that allows clinicians to continuously monitor all core vital signs in a wearable and noninvasive way. The device is intended for use in a clinical setting as vital sign data are sent to a central nurse monitoring station within the hospital setting. A number of similar wearable products are available to monitor other vital sign information. For example, [7] presents a wearable photoplethysmography (PPG) sensor that enables continuous monitoring of vital signs such as oxygen saturation, RR, heart rate and blood pressure. The small wearable device enables users to store all the measured data and send it to a remote location. In [8] a different style of wearable sensor in the form of in-ear sensors was developed which measures the ballistocardiogram (BCG) (a measure of ballistic forces on the heart), ECG and PPG (a volumetric measurement of an organ) in order to monitor stroke volume (SV), pulse transit time (PTT), pre-ejection period (PEP) and cardiac output (CO). There have also been efforts to include the wearable sensors within fabric so that the user is not irritated when wearing a sensor and also to prevent a user from forgetting to wear the sensor on a daily basis. For example, [9] presents a sensor that incorporates a microwave stethoscope [10] which is capable of reading of heart beat, RR, SV and changes in lung water contents. In [11] a wearable sensor is developed with the aim that the user would not be constricted in any way whilst wearing it. The authors are now working on a "bionic skin" type wearable sensor that sits on top of our skin and will eventually be capable of continuously monitoring vital signs. However, all of these styles of sensors require the users to be wearing them in order for their vital signs to be assessed. Common methods used for the measurement of BPM and RR include the use of the Doppler effect (e.g. [12]-[16]) or vision-based techniques (e.g. [17], [18]). However, the majority of radar-based methods can have inaccuracies, particularly in the measurement of the heart rate due to several spurious peaks being produced at harmonics and intermodulation frequencies [19]. Furthermore, vision-based methods for heart rate measurements are highly accurate when good quality vision data are available. However, other sensors such as sound and tactile are required for use in areas where vision performs poorly. The use of these other sensors will enable collection of data that vision systems will not be able to measure, such as vibration and thermal data from physical contact. Interestingly, to date, force or tactile sensing has not been heavily researched for use in the measurement of human vital signs. One previous example used a range of force sensors such as multi-point polymer force/pressure sensors [20], Polyvinylidene Fluoride (PVDF) tactile array sensors [21] and piezoresistive tactile sensors [22] to measure BPM. Kerr et al., have developed algorithms to measure BPM, RR [23] and CRT using a BioTAC tactile sensor together with a RGB camera [24], and this will be discussed in Section II. A further alternative approach to vital sign monitoring would be the use of remote monitors fixed to beds or other items. For example, a bedbased system that monitors the person's pulse and breathing rate via force sensitive resistors, microphones, etc. [25]. Also [26] uses an optical fibre interferometer placed in a mattress to continuously measure a person's vital signs overnight. A third-order low bandpass filter was applied to the data collected from the sensor and further bandpass filters ranging in cut-off frequencies were used to detect BPM and RR. Accuracies of approximately $95.7 \%$ for heartbeat and $93.8 \%$ for respiration were achieved using datasets from five males with 60 seconds of data collection. The authors continued this research in later work to test the method on a user during a full night's sleep over a period of 9.75 hours [27].

However, all of the measurement techniques and devices require either (or both) the wearing or fitting of a device in order to take measurements, and the expertise of a trained professional. They fail to deal with situations in which a medical professional is not present, where the patient is not currently wearing the measuring device, or if the patient is in a medical emergency situation that prevents them from operating a vital signs measuring device. Some previous ambient living or robot-based projects have tried to address some of these issues individually; the EMERGE project [28] focussed on detecting and counteracting emergencies using ambient and unobtrusive sensors to provide early warning of emergencies. Somewhat more closely aligned with the goal of obtaining vital signs information was the NurseBot project [29] that developed a mobile robotic assistant for monitoring and providing reminders. The robot was equipped with standard medical grade vital signs monitoring equipment that required the user (or medical professional) to attach the equipment and then data are transmitted to medical professionals in another location. The CAALYX project monitors a person's vital signs through the use of a "smart shirt" - a wearable smart device 
(LifeShirt) [30], [31]. Gecko Systems' CareBot is a personal robot equipped with multiple vital sign sensors that can follow an elderly person in their home. It can carry on-board thirdparty sensors for monitoring blood pressure, pulse, oxygenation levels and blood sugar levels - again, however, these systems all require some user interaction in order to use the vital sign monitoring equipment. The CareBot is also capable of verbal interaction, the delivery of medicine, video monitoring, and two-way interactions. This remote two-way interaction approach (telepresence) has also been used to obtain vitals by facilitating remote doctor-patient interactions, again requiring the patient to operate monitoring equipment. This paper details an approach to provide the capability to autonomously measure an individual's vital signs and instantly communicate its findings to the relevant carer, medical or emergency services personnel using a novel visualisation method to clearly communicate the information.

Cloud computing can provide several benefits to all the stakeholders in the healthcare ecosystem (patients, providers, payers, etc.). In [32] an effective healthcare monitoring system was developed and implemented based on an Internet of Things (IoT). The proposed system monitors the vital health parameters and transmits the data using wireless communication, which is then further transferred to a network via a Wi-Fi module. The data can be accessed at any time, promoting the reception of the current health status of the patient. In the case of abnormal behaviour or any vital signs which are not recognised, a carer as well as medical professionals are notified immediately through a message service or an audio signalling device (buzzer).

Vital signs data are quite different from other types of data used in enterprise systems in that they do not have a structure in the form of a database (DB) repository, but rather a single data type [33]. This is because it is much more effective to access vital signs data stored in the form of a file than as a DB. For this reason, the vital signs data were represented in the form of an XML document, which is meta-model-based and a module is developed to check the state of the vital signs. In this manner, one can achieve interoperability, because the system uses a standardised way of representing the vital signs data and a standardised specification language. This approach is easily adaptable to service content that changes rapidly and reduces the maintenance costs, through the use of $\mathrm{OCL}$, a textbased specification language, rather than a programming language. A cloud-based approach for the design of interoperable electronic health record (EHR) systems is presented in [34]. It can achieve semantic interoperability through the use of a generic design methodology which uses a reference model that defines a general-purpose set of data structures and an archetype model that defines the clinical data attributes. Compared with client-server-based EHR systems, the cloud-based system can achieve better performance in such fields as interoperability, scalability, maintainability and portability. With the support of mobile cloud computing, [35] declared that wireless area networks can be significantly enhanced for massive deployment of pervasive healthcare applications. Vital sign data are transmitted to the cloud using energy-efficient routing, cloud resource allocation, semantic interactions, and data security mechanisms. A Health-loT-enabled monitoring framework is presented in [36], where ECG and other healthcare data are collected by mobile devices and sensors and securely sent to the cloud for seamless access by health and social care professionals. Signal enhancement, watermarking, and other related analytics can be used to avoid identity theft or clinical error by health and social care professionals. To provide a more convenient service and environment for healthcare, [37] proposes a cyber-physical system for patient-centric healthcare applications and services, called Health-CPS, built on cloud and big data analytics technologies. This system consists of a data collection layer with a unified standard, a data management layer for distributed storage and parallel computing, and a data-oriented service layer. Cloud computing can enhance the performance of the healthcare system so humans can enjoy various smart healthcare applications and services.

The structure of this paper is as follows. Section II presents an overview of how vital sign data are recorded and processed. Section III details how Cloud based processing and storage of the collected data are completed. Section IV outlines the data analysis visualisation proposed for multiple users' health status. Finally, the paper is concluded, and an outline of future work is discussed in Section V.

\section{ROBOt-BASED VitAL SIGNS MONITORING}

We have developed and evaluated algorithms for determining a human's BPM, Pulse to Pulse Interval (PPI), RR and Breath to Breath Interval (BBI) via tactile sensing using a robotic system comprised of a five-fingered Shadow Hand [38] with three BioTAC sensors [39]. The Shadow Hand (shown in Figure 2) has 21 degrees of freedom and has similar dexterity to a human hand. the BioTAC ${ }^{\text {тм }}$ biomimetic tactile sensor achieves tactile perceptions similar to humans using a fingershaped sensor array that provides simultaneous information about contact forces, microvibrations and thermal fluxes induced by contact with external objects [40, 41]. A cross section view of the BioTAC fingertip tactile sensor is shown in Figure 1 .

There are various sensors on the fingertip that enable retrieval of the contact forces, microvibrations and thermal flux sensory information. An array of 19 electrodes measures the force applied on the fingertip by reading the impedance between each electrode and four common excitation electrodes. Inside the rigid core of the fingertip there is a pressure sensor. This sensor reads pressure changes in the conductive fluid located between the hard core and the elastomeric skin, following microvibrations which are caused when the fingerprint on the elastomeric skin makes contact 
with other objects. Additionally, thermal data can be collected, made possible by the thermistor which is located in the tip of the fingertip.

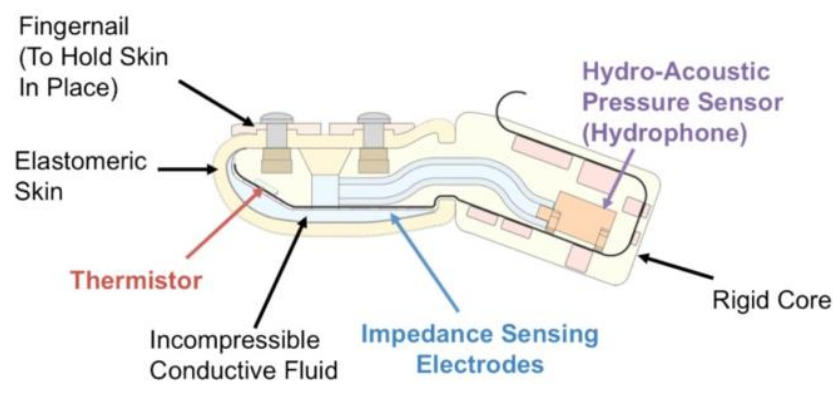

Figure 1: Image showing a cross-sectional view of the BioTAC fingertip [39].

The BPM, RR and CRT of 12 healthy human subjects were measured by analysing data collected from their wrists, chest and forehead respectively. Vibration data from the wrist of each subject were collected to determine the vibration (of the internal conductive fluid of the BioTAC) caused by the subjects' pulse when the BioTAC fingertip was pressed against the radial artery. To achieve this, the Shadow Hand is positioned such that the thumb is below the subject's wrist. The Shadow Hand then moves its first finger and middle finger in small increments towards the radial artery on the ventral aspect of the thumb side of the subject's wrist, as illustrated in Figure 2(a). The electrodes in the BioTAC fingertip are used to constantly measure the force applied. Once the Shadow Hand's first and middle finger have made sufficient contact with the wrist, the fingers stop moving and recording of the vibration data begins. Figure 2(a) illustrates how this action replicates the human action of measuring a subject's pulse by pressing fingers on the wrist and counting the number of beats per minute. This method can determine whether a person has a slow heart rate $(<60 \mathrm{BPM})$, i.e. bradycardic, a normal heart rate $(60-100$ BPM) or a fast heart rate (> 100 BPM), i.e. tachycardic.

It is also possible to determine if a person has a slow, or normal RR using the same robotic system. Contact with the chest of the human subject was made using the Shadow Hand, as shown in Figure 2(b). The hand is initialised in an open position and then both the first and middle finger are moved towards the upper left side of the subject's chest in small increments. The electrodes on the BioTAC were constantly monitored until sufficient contact was deemed to have been made with the subject's chest. Breathing can cause artefacts and noise when recording from the BioTAC fingertip so both vibration data and thermal conductivity data were collected. During a breathing motion the subject's chest wall rises and the subject's skin presses against the inside of their clothing resulting in concentrated indirect contact with the skin. The
BioTAC thermal conductivity value fluctuates as the fingertip senses the change in temperature and friction during the breathing motion. The method presented here was validated against readings obtained by a medical professional on the same subjects and the developed approach mimics the procedure carried out by the medical professional.

An approach to measure CRT was also developed using the pressing action of the robot fingertip and artificial vision to analyse changes in skin colour. As discussed, CRT is the time taken for the subject's natural colour to restore in an external capillary bed following blanching caused by pressure being applied. Here, approximately 5 seconds of video data are recorded in a region of interest (approximately $12 \mathrm{~cm}^{2}$ ) on the subject's forehead. The first finger of the Shadow Hand, which is equipped with a small RGB camera, is then pressed against the centre of the subject's forehead for 5 seconds with a constant, painless force. The fingertip is then moved back to the starting position and video data in the region of interest on the forehead is captured for a further 10 seconds.

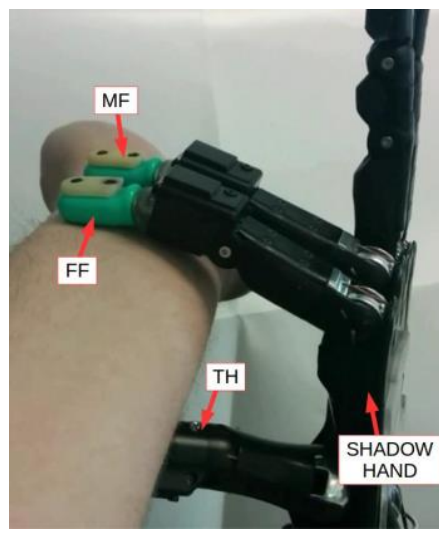

(a)

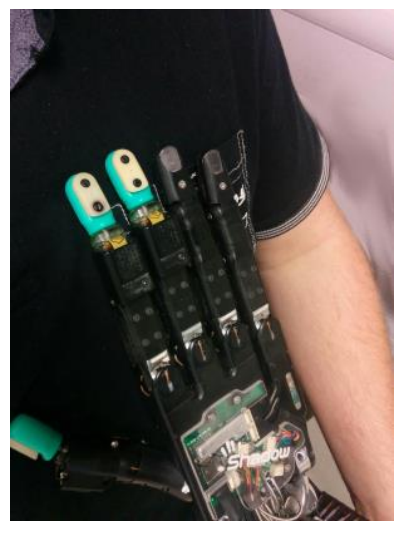

(b)
Figure 2: (a) Image showing the Shadow Hand taking the subject's pulse; (b) Image showing the Shadow Hand measuring a subject's $\mathrm{RR}$ by resting on the subject's chest

There is a short period of time before the fingertip makes contact with the subject's forehead which enables the camera to capture the subject's natural skin colour. Pressure is then applied to the subject's forehead using the finger which causes the skin to blanch. The time taken for the blanched skin to return to its natural colour is then calculated; this is the CRT. The force at which the fingertip presses against the subjects' forehead is regulated and the change in colour is determined. This procedure replicates the procedure carried out by medical professionals when measuring CRT. The methods presented here for determining BPM, RR and CRT all maintain anonymity of the subject.

\section{Cloud-Based Computation And Storage}

For data processing and storage, we build a structure based on the producer/consumer model. When data are produced, they can be considered as the products made by workers in a 
factory. Therefore, a method is needed to transfer the products. We use message queue RabbitMQ [42] as the data buffer module to assure the data created by the producers can be consumed and a signal is fed back. Furthermore, by setting up a related mechanism for message queues, we can adopt the consuming strategy with time as the baseline.

At the end of consumer stage, an algorithm is used to process the data from the message queue. This mainly consists of the classification of time, geographical area and person, along with the judgement of an abnormal alarm. When the consumers are fulfilling these tasks, the data are processed fully in parallel. Different consumers are responsible for different data so that the system efficiency can be improved. When these steps are finished, data persistence will be implemented in the database. Here, we used MongoDB [43] whose data structure is made of key-value pairs. In MongoDB, data are similar to JSON objects and easily visualised for the interface. For high load situations, MongoDB can ensure the system performance by increasing the node quantity. For data transmission to the cloud, the whole procedure is implemented in a private network. A VPN (Virtual Private Network) server is built to ensure the security of data transmission. Figure 3 shows the architecture of data processing in the cloud.

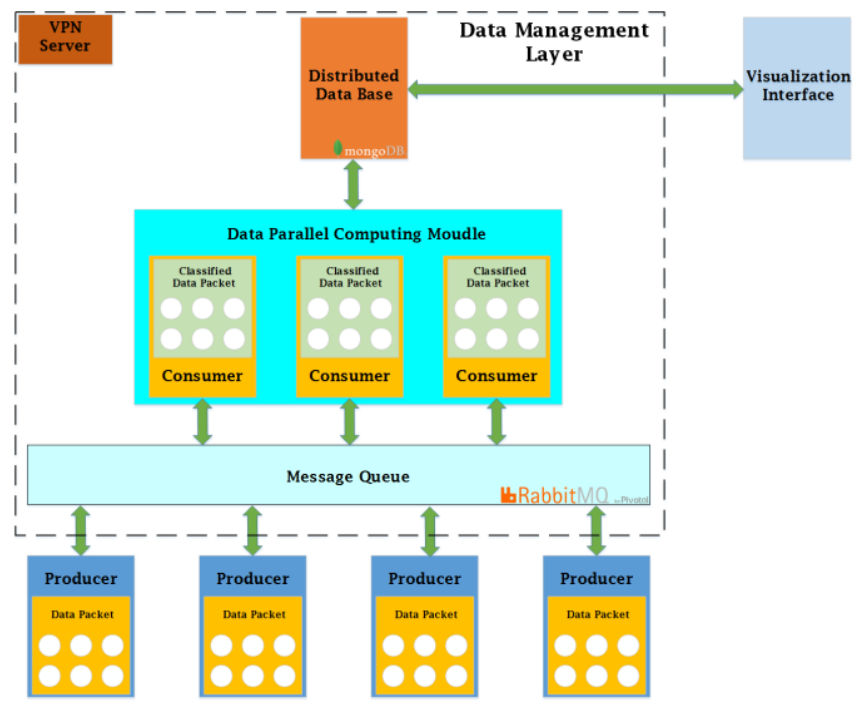

Figure 3: The architecture of data processing in the cloud

\section{Vital Signs data Visualisation}

In order for a medical professional to be able to view the current health status of multiple service users, a novel method to visualise the collected and assessed vital sign data is required. To achieve an overall view of the health status for multiple service users in each region of a country (in this case China), we propose a regional map with an overlay of colourcoded markers which will indicate the health status of each user or group of users within an elderly persons nursing home for example. Figure 4 shows an example of such a map for the Liaoning Region situated in the north east of China. A green marker on the map indicates that the users' health status is within the expected boundaries for a healthy adult with respect to their BPM, RR and CRT. An amber marker indicates that at least one of the service users' vital signs is slightly outside of the expected boundaries (i.e. either $10 \%$ over or under the expected range of BPM and/or RR). A red marker indicates that at least one of the service users' vital signs is currently being, or has been, recorded as being outside of the expected range for a healthy adult.

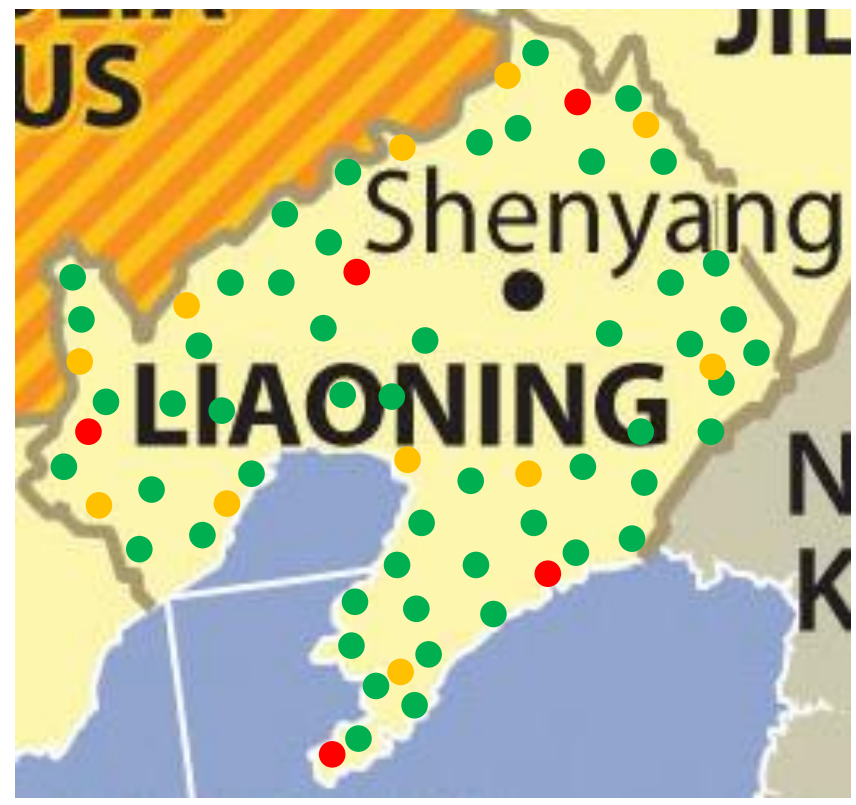

Figure 4: Map showing an overview of service users' health status for the Liaoning Region of China

The regional map in Figure 4 is interactive and therefore a medical professional can click on each marker for further information as they review the region. Clicking on the marker will open a further graph with more detail about the particular service user, illustrated in Figure 5. The chart in Figure 5 shows the current reading and the five previous observations (medical history) for each of the three vital signs. This allows the medical professional to view a brief history of the service user's health status and ascertain if they require further assessment or assistance. In order to get a more detailed insight into the services users' vital signs, the medical professional can click on any of the three vital signs in the radar chart shown in Figure 5 which will load a live graph of that vital sign which can be used to view the ongoing measurement of the vital sign as well as historical observations. An example of the graph available for BPM is shown in Figure 6.

The visualisation maps and graphs in Figure 4, Figure 5 and Figure 6 allow a medical professional to obtain an initial overview of many service users and then focus attention on any individual service users which demonstrate deteriorating health. 


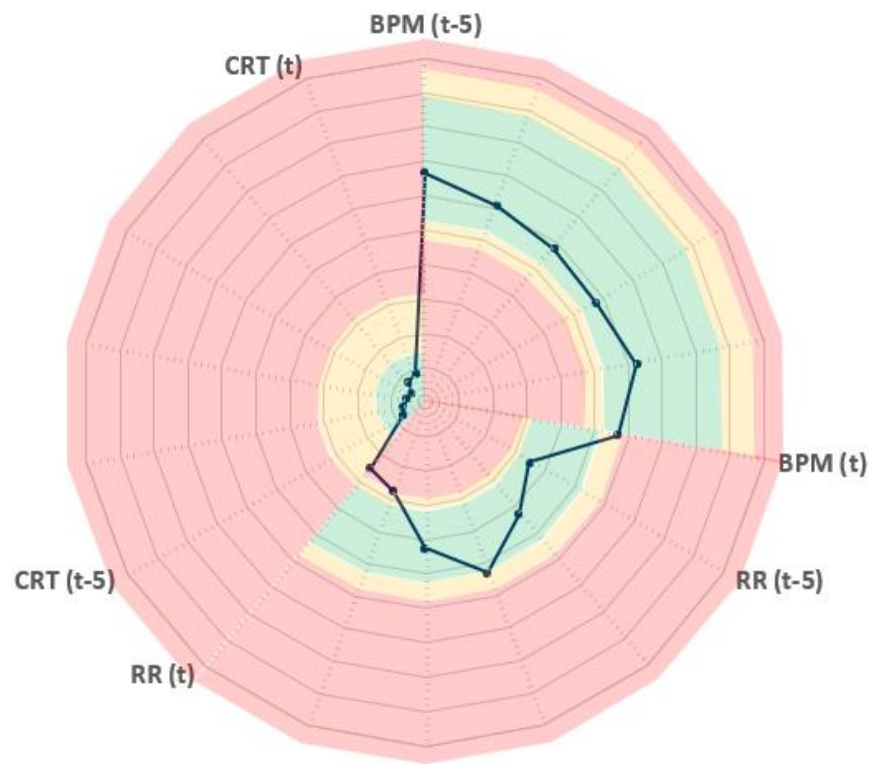

Figure 5: Radar chart showing detail on three assessed vital signs for a particular service user. The chart shows the current reading (t) and five previous readings for BPM, RR and CRT (back to t-5).

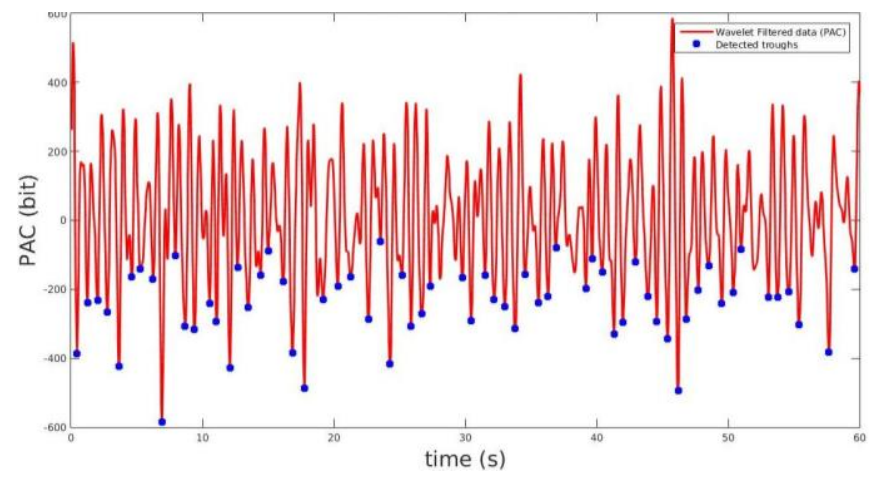

Figure 6: A graph showing the detect heart beats of a service user over a one-minute time interval

\section{CONCLUSION AND FUTURE WORK}

Being able to ascertain a human's health status by measuring their vital signs remotely is a positive step towards elderly persons living at home independently for longer. Furthermore, being able to quickly and effectively review multiple service users' current health status would be extremely beneficial in rural areas globally. The use of the Cloud for processing and storing the data means that 'big' vital signs data can be stored and reviewed by a medical professional. This paper outlines a cloud-based processing and storage architecture that can process vital signs data collected from multiple service users. Moreover, an effective visualisation tool is presented that will assist a medical profession in the fast analysis of multiple service users' health status. Future work will include the use of additional machine learning algorithms to learn from false positives or false negatives with regards to identifying service users' to further investigate based on their three assessed vital signs.

\section{ACKNOWLEDGEMENTS}

The collaborations and research outlined in this paper have been funded by Ulster University's GCRF pump-priming funding.

\section{REFERENCES}

[1] WHO, "No Title," World Health Organisation, 2015. [Online]. Available: http://www.who.int/mediacentre/factsheets/fs404/en/.

[2] UN, “World Population Ageing," 2015.

[3] J. Hu et al., "An advanced medical robotic system augmenting healthcare capabilities - robotic nursing assistant," in 2011 IEEE International Conference on Robotics and Automation, 2011, pp. 6264-6269.

[4] G. Stollnberger et al., "User requirements for a medical robotic system: Enabling doctors to remotely conduct ultrasonography and physical examination," in 2016 25th IEEE International Symposium on Robot and Human Interactive Communication (RO-MAN), 2016, pp. 1156-1161.

[5] J. Beckow, "The truth about capillary refill.," JEMS a J. Emerg. Med. Serv., vol. 30, no. 1, p. 14--discussion, 2005.

[6] K. S. Strozik, C. H. Pieper, and J. Roller, "Capillary refilling time in newborn babies: normal values," Arch. Dis. Child. - Fetal Neonatal Ed., vol. 76, no. 3, pp. F193--F196, 1997.

[7] V. Mouradian, A. Poghosyan, and L. Hovhannisyan, "Continuous wearable health monitoring using novel PPG optical sensor and device," in 2014 IEEE 10th International Conference on Wireless and Mobile Computing, Networking and Communications (WiMob), 2014, pp. 120123.

[8] D. D. He, E. S. Winokur, and C. G. Sodini, "An Ear-Worn Vital Signs Monitor," IEEE Trans. Biomed. Eng., vol. 62, no. 11, pp. 2547-2552, Nov. 2015.

[9] R. Gagarin, G. C. Huang, A. Rabbi, and M. F. Iskander, "Textile sensor for monitoring vital signs," in 2014 IEEE Antennas and Propagation Society International Symposium (APSURSI), 2014, pp. 965-966.

[10] R. Gagarin, N. Celik, G. C. Huang, and M. F. Iskander, "Microwave Stethoscope, a new noninvasive multiple vital signs sensor: Human clinical trials," in Proceedings of the 2012 IEEE International Symposium on Antennas and Propagation, 2012, pp. 1-2.

[11] T. Someya, "Building bionic skin," IEEE Spectr., vol. 50, no. 9, pp. 50-56, Sep. 2013.

[12] J. C. Lin, "Noninvasive microwave measurement of respiration," Proc. IEEE, vol. 63, no. 10, p. 1530, Oct. 1975.

[13] J. H. Choi and D. K. Kim, "A Remote Compact Sensor for the Real-Time Monitoring of Human Heartbeat and Respiration Rate," IEEE Trans. Biomed. Circuits Syst., vol. 3, no. 3, pp. 181-188, Jun. 2009.

[14] G. Lu et al., "Contact-Free Monitoring of Human Vital Signs via a Microwave Sensor," in 2011 5th International Conference on Bioinformatics and Biomedical Engineering, 2011, pp. 1-3.

[15] B. Iyer, A. Kumar, N. P. Pathak, and D. Ghosh, "Concurrent multi-band RF system for search and rescue of human life during natural calamities," in IEEE MTT-S International Microwave and RF Conference, 2013, pp. 1-4.

[16] A. Boothby et al., "Accurate and continuous non-contact vital signs monitoring using phased array antennas in a clutter-free anechoic chamber," in 2013 35th Annual International Conference of the IEEE Engineering in Medicine and Biology Society (EMBC), 2013, pp. 28622865.

[17] H. E. Tasli, A. Gudi, and M. den Uyl, "Remote PPG based vital sign measurement using adaptive facial regions," in 2014 IEEE International Conference on Image Processing (ICIP), 2014, pp. 1410-1414.

[18] H.-Y. Wu, M. Rubinstein, E. Shih, J. Guttag, F. Durand, and W. T. Freeman, "Eulerian Video Magnification for Revealing Subtle Changes in the World," ACM Trans. Graph. (Proceedings SIGGRAPH 2012), vol. 31, no. 4, 2012

[19] L. Ren, H. Wang, K. Naishadham, Q. Liu, and A. E. Fathy, “Non-invasive 
detection of cardiac and respiratory rates from stepped frequency continuous wave radar measurements using the state space method," in 2015 IEEE MTT-S International Microwave Symposium, 2015, pp. 1-4.

[20] X. Mi and F. Nakazawa, "A multipoint thin film polymer pressure/force sensor to visualize traditional medicine palpations," in IEEE SENSORS 2014 Proceedings, 2014, pp. 843-846.

[21] Y. Qian, A. Song, and R. Yan, "Design and realization of an array pulse detecting tactile sensor," in 2011 IEEE International Instrumentation and Measurement Technology Conference, 2011, pp. 1-5.

[22] C. M. Chang, F. W. Lee, Y. H. Lin, and Y. J. Yang, "Tunneling piezoresistive tactile sensing array for continuous blood pressure monitoring," in 2016 IEEE 29th International Conference on Micro Electro Mechanical Systems (MEMS), 2016, pp. 169-172.

[23] E. Kerr, T. M. McGinnity, S. Coleman, and A. Shepherd, "Towards Pulse Detection and Rhythm Analysis using a Biomimetic Fingertip," in 2015 IEEE International Joint Conference on Neural Networks, July 12-17 2015, Killarney, Ireland, 2015.

[24] E. Kerr, T. M. McGinnity, and S. Coleman, "Tactile Sensing for Assistive Robotics," Ulster University, 2018

[25] A. Bartels and D. Harder, "Non-invasive determination of systolic blood pressure by heart sound pattern analysis," Clin. Phys. Physiol. Meas., vol. 13, no. 3, pp. 249-256, 1992.

[26] S. Šprager, D. Donlagić, and D. Zazula, "Monitoring of basic human vital functions using optical interferometer," in IEEE 1Oth INTERNATIONAL CONFERENCE ON SIGNAL PROCESSING PROCEEDINGS, 2010, pp. 1-4.

[27] S. Šprager, D. Donlagić, and D. Zazula, "Overnight heartbeat monitoring by using unobtrusive optical interferometric measurements," in 2012 IEEE-EMBS Conference on Biomedical Engineering and Sciences, 2012, pp. 847-852.

[28] EC, “EMERGE: Emergency Monitoring and Prevention." p. Project No. 045056.

[29] J. Pineau, M. Montemerlo, M. Pollack, N. Roy, and S. Thrun, "Towards robotic assistants in nursing homes: Challenges and results," in Robotics and Autonomous Systems, 2003, vol. 42, no. 3-4, pp. 271-281.

[30] C. Gopalsamy, S. Park, R. Rajamanickam, and S. Jayaraman, "The wearable motherboard ${ }^{\mathrm{TM}}$ : The first generation of adaptive and responsive textile structures (ARTS) for medical applications," Virtual Real., vol. 4, no. 3, pp. 152-168, 1999.

[31] H. F. Machiel Van der Loos, N. Ullrich, and H. Kobayashi, “Development of sensate and robotic bed technologies for vital signs monitoring and sleep quality improvement," Auton. Robots, vol. 15, no. 1, pp. 67-79, 2003.

[32] S. Chaudhury, D. Paul, R. Mukherjee, and S. Haldar, "Internet of Thing based healthcare monitoring system," in 2017 8th Industrial Automation and Electromechanical Engineering Conference, IEMECON 2017, 2017, pp. 346-349.

[33] T.-W. Kim and H.-C. Kim, "Service-oriented architecture structure for healthcare systems utilising vital signs," IET Commun., vol. 6, no. 18, pp. 3238-3247, 2012.

[34] A. Bahga and V. K. Madisetti, "A cloud-based approach for interoperable electronic health records (EHRs)," IEEE J. Biomed. Heal. Informatics, vol. 17, no. 5, pp. 894-906, 2013.

[35] J. Wan, C. Zou, S. Ullah, C.-F. Lai, M. Zhou, and X. Wang, "Cloud-enabled wireless body area networks for pervasive healthcare," IEEE Netw. Mag., vol. 27, no. 5, pp. 56-61, 2013.

[36] M. S. Hossain and G. Muhammad, "Cloud-assisted Industrial Internet of Things (IloT) - Enabled framework for health monitoring," Computer Networks, 2015

[37] Y. Zhang, M. Qiu, C. W. Tsai, M. M. Hassan, and A. Alamri, "Health-CPS: Healthcare cyber-physical system assisted by cloud and big data," IEEE Syst. J., vol. 11, no. 1, pp. 88-95, 2017.

[38] Shadow Robot Company, "Shadow Dexterous Hand." 2017.

[39] Syntouch, "The Syntouch Website." 2013.

[40] T. Yamamoto, N. Wettels, J. A. Fishel, C.-H. Lin, and G. E. Loeb, "BioTac Biomimetic Multi-modal Tactile Sensor," J. Robot. Soc. Japan, vol. 30, no. 5, pp. 496-498, 2012.

[41] C.-H. Lin, T. W. Erickson, J. A. Fishel, N. Wettels, and G. E. Loeb, “Signal processing and fabrication of a biomimetic tactile sensor array with thermal, force and microvibration modalities.," in ROBIO, 2009, pp. 129134.

[42] "RabbitMQ[EB/OL]." [Online]. Available: http://www.rabbitmq.com/.

[43] "MongoDB[EB/OL]." [Online]. Available: https://www.mongodb.com/. 